\title{
Stability studies of polypyrole- derived carbon based symmetric supercapacitor via potentiostatic floating test
}

\author{
A. Bello, F. Barzegar, M. J. Madito, D. Y. Momodu, A. A. Khaleed, T. M. Masikhwa, J. K. \\ Dangbegnon and N. Manyala
}

Department of Physics, Institute of Applied Materials, SARCHI Chair in Carbon Technology and Materials, University of Pretoria, Pretoria 0028, South Africa.

Corresponding Author: *Email address: Ncholu.Manyala@up.ac.za (N. Manyala)

\section{Highlights}

- Synthesis of activated carbon (AC) from the carbonisation of polypyrole (PPY).

- $\mathrm{KOH}$ activation of the $\mathrm{AC}$ to produce porous carbons.

- Symmetric device based on the porous AC exhibit good electrochemical performance.

- Supercapacitor show excellent stability based on potentiostatic floating test for $200 \mathrm{~h}$

\begin{abstract}
This work present the synthesis and electrochemical performance of mesoporous carbon material derived from the activation and carbonisation of polypyrole (PPY). Electrochemical performance of the PPY derived carbon was investigated in a two electrode cell in a $6 \mathrm{M}$ $\mathrm{KOH}$ alkaline electrolyte. The symmetric device was subjected to floating test (voltage holding) for $\sim 200 \mathrm{~h}$ at a voltage of $1.2 \mathrm{~V}$ and the specific capacitance as well as the resistance of device was determined after every $10 \mathrm{~h}$ of floating. The device exhibited a specific capacitance of $179 \mathrm{~F} \mathrm{~g}^{-1}$ at $0.5 \mathrm{~A} \mathrm{~g}^{-1}$ and $158 \mathrm{~F} \mathrm{~g}^{-1}$ at $10 \mathrm{~A} \mathrm{~g}^{-1}$. In addition, after floating for $\sim 120 \mathrm{~h}$ the resistance was constant with a very slow decrease in capacitance.
\end{abstract}


Beyond $\sim 120 \mathrm{~h}$, a quick decrease in capacitance was observed with a corresponding increase in resistance, indicating a possible deterioration to the electrodes. Remarkably, at the end of floating, the specific capacitance calculated at $5 \mathrm{~A} \mathrm{~g}^{-1}$ was $120 \mathrm{~F} \mathrm{~g}^{-1}(\sim 25 \%$ decay in capacitance from the initial value $160.5 \mathrm{~F} \mathrm{~g}^{-1}$ at $5 \mathrm{~A} \mathrm{~g}^{-1}$ ) suggesting good stability of the device over a long period of time ( $~ 8$ days).

Keywords: Polypyrole, activated carbon, supercapacitor, potentiostatic floating, aqueous electrolyte.

\section{Introduction}

Supercapacitor is an electrochemical capacitor that stores charges using non-faradaic process (electrostatically at the interface of the electrodes) which gives rise to a potential dependent storage ability known as the electric double (EDLC), as well as the faradaic charge storage mechanism (charge transfer between the electrodes similar to ion intercalation-deintercalation giving rise to unique potential charge phenomenon often referred to as pseudocapacitance [1-3]).

Supercapacitors (EDLC) have capacitance far greater than that obtained in conventional dielectric capacitors with their existing benefits of high power density and long cycle life [3]. Thus, making them appropriate for a wide range of potential applications, such as in renewable power generation systems, power backup systems and transient energy saving application such as energy capture during braking in vehicles, in construction equipment such as cranes and for opening of doors in the A380 Jumbo jet [4]. In a general term, a symmetric EDLC consist of carbon based materials as both positive and negative electrodes, 
a separator and an electrolyte which is suitable to the porosity of the carbon material been investigated. Basically, the choice of carbon as electrodes for EDLC is due to their excellent electrical conductivity and high specific surface area (SSA) since the capacitance linearly increases with the SSA as $C=\varepsilon_{r} \varepsilon_{0} A / d$, where $C$ is the capacitance measured in Farad, $\varepsilon_{r}$ is the dielectric constant of the electrolyte, $\varepsilon_{0}$ is the dielectric constant of the vacuum, $d$ is the charge separation distance and A is the SSA of the electrode [2].

Many forms of carbon material have been trialled as electrodes for EDLC. For example, carbide-derived carbons [5,6], graphene [7,8], onion rings [2,9] activated carbon [10], carbon nanotubes (CNTs) [11,12], and carbon aerogels [13] have been tested for EDLC applications. Nevertheless, amongst these different carbon materials, activated carbon is the only one that is been used commercially using organic solutions as electrolyte $(2.7 \mathrm{~V})$ [14], while others are still being extensively researched. Organic electrolyte have extended voltage window which is beneficial for high energy, nonetheless, they suffer from low electrical conductivity, toxicity and high cost when compared with aqueous electrolytes (acidic, alkaline or neutral) that present better conductivity and are less expensive $[14,15]$. Though, aqueous electrolytes (alkaline and acidic) are limited in voltage (up to $1.23 \mathrm{~V}$ ), due to the thermodynamics decomposition of water, neutral electrolytes are able to achieve higher voltage than the alkaline and acidic aqueous electrolytes. For example symmetric supercapacitor operating at $1.6 \mathrm{~V}$ in neutral $1 \mathrm{M}$ and $0.5 \mathrm{~mol} \mathrm{~L}^{-1} \mathrm{Na}_{2} \mathrm{SO}_{4}$ media were reported [16,17]. Sulphate solutions including $1 \mathrm{~mol} \mathrm{~L}^{-1} \mathrm{Li}_{2} \mathrm{SO}_{4}, \mathrm{Na}_{2} \mathrm{SO}_{4}$ and $\mathrm{K}_{2} \mathrm{SO}_{4}$ were examined as electrolyte for supercapacitors by Fic et. al.. In their work the metal cations were characterised by their ion-solvent diameter and the solvation/desolvation energy. They concluded that $1 \mathrm{~mol} \mathrm{~L}^{-1} \mathrm{Li}_{2} \mathrm{SO}_{4}$ electrolyte is the most promising amongst the three 
electrolytes investigated due to its strong solvation of ions that is beneficial for the wide operational voltage range up to $2.2 \mathrm{~V}[18]$.

The appropriate choice of electrolyte and electrode material porosity plays important roles in determining the overall performance of the EDLCs devices (rate capability), thus, porous graphitic carbons, exhibiting three-dimensional (3D) framework and nitrogen doped mesoporous carbon have become attractive because of their high inherent electrical conductivity and 3D architecture that could be beneficial for enhancement of EDLCs devices $[14,19,20]$.

Furthermore, the evaluation of supercapacitor devices performance (capacitance and resistance) is mostly based on cyclic voltammetry (CV), constant current charge-discharge (CCCD) and electrochemical impedance spectroscopy (EIS). Apart from these measurements, the stability of supercapacitors is a feature of major importance. The traditional method of evaluating the stability is via the constant current charge-discharge (CCCD) cycling over several thousands of cycles and these data are usually presented in the literature with excellent stability and no significant capacitance loss showing no degradation of the electrodes $[21,22]$. Voltage-holding (or floating) test recently has been established as an alternative and reliable method for stability analysis of supercapacitor electrodes [23,24]. The floating test is performed at a constant load by holding the device at the highest voltage and calculating the capacitance over the entire period shown in scheme 1 this sequence is usually repeated several times. 


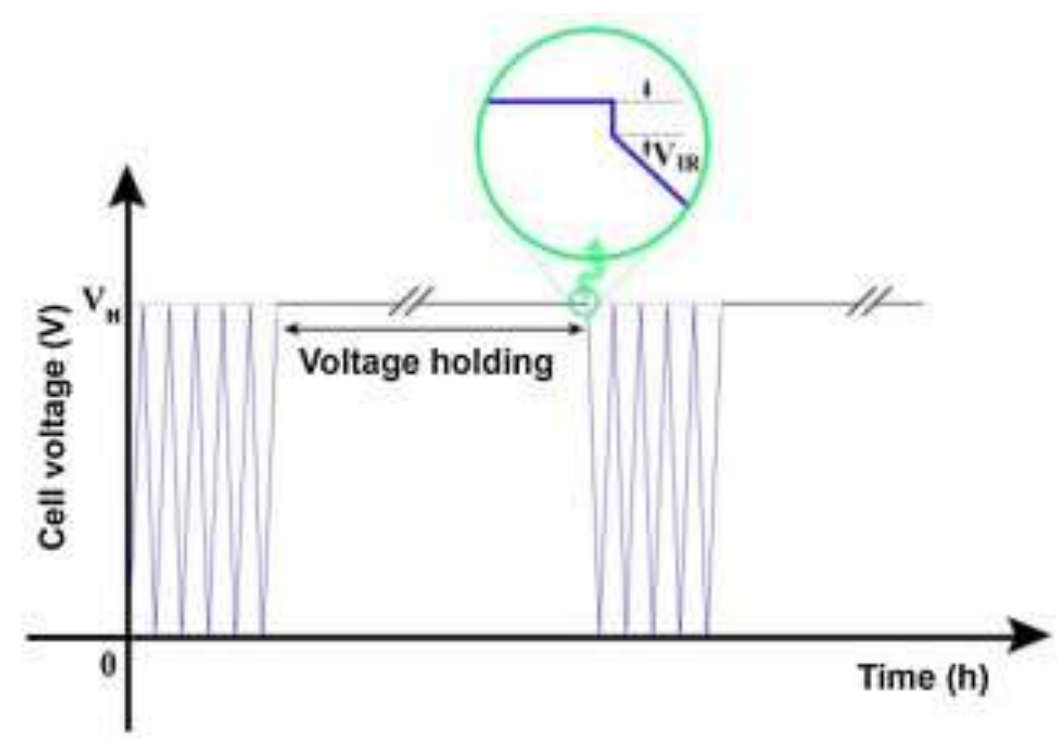

Scheme 1 Schematic illustration of the floating test experiment

One great advantage of floating is that its gives a direct insights into the possible effect and degradation phenomena that might occur during the electrochemical process in EDLCS devices at elevated cell voltages when compared with the traditional cycling test which often times shows no degradation. It also gives a true resistance effect after subjecting to maximum voltage which will be close to practical relevance, thus, it is important to study different carbon materials from different sources under such conditions to ascertain the actual electrochemical performance over a long period of time and link the electrochemical performance with the physicochemical changes that might occur during the electrochemical process. Thus, herein, we report on synthesis of low-cost porous graphitic carbon networks exhibiting high surface area with a three dimensional interconnected porous architecture using conducting polymer as the starting precursor. The process starts with the cross-linking and polymerization of a polymeric monomer into three dimensional structures, followed by chemical activation and carbonization into porous carbon by thermal annealing. Furthermore, the electrochemical performance of the porous carbon electrodes and the 
stability was studied in detail based on floating in $6 \mathrm{M} \mathrm{KOH}$ aqueous. Remarkably, the device showed good stability after floating for $190 \mathrm{~h}$ with a capacitance decay of $25 \%$.

\section{EXPERIMENTAL}

\subsection{Synthesis of polypyrole (PPY)}

PPY was synthesised as follows: $2.4 \mathrm{~g} \mathrm{FeCl}_{3}$ was dissolved in $50 \mathrm{ml}$ deionized water at room temperature and stirred for $30 \mathrm{~min}$ followed by the slowly addition of $0.5 \mathrm{ml}$ of Pyrole monomer with continuous stirring for $12 \mathrm{~h}$. This process leads to the following reaction

$\mathrm{nC}_{4} \mathrm{H}_{4} \mathrm{NH}+2 \mathrm{FeCl}_{3} \rightarrow\left(\mathrm{C}_{4} \mathrm{H}_{2} \mathrm{NH}\right)_{\mathrm{n}}+2 \mathrm{FeCl}_{2}+2 \mathrm{HCl}$

After the synthesis, the obtained product was washed continuously to remove the $\mathrm{FeCl}_{2}$ and $\mathrm{HCl}$ present in the sample. Afterwards, the sample was dried in an electric oven at $80^{\circ} \mathrm{C}$ for $24 \mathrm{~h}$.

\subsection{Synthesis of porous carbon (AC)}

The obtained dried PPY powder was mixed chemically with potassium hydroxide (KOH) pellets in a 1:4 weight ratio [25]. The mixture was then positioned at the centre of a horizontal tube furnace annealed for $1 \mathrm{~h}$ at $800{ }^{\circ} \mathrm{C}$ with a ramp of $5{ }^{\circ} \mathrm{C} /$ minute under nitrogen flow to increase the surface area. The activated carbon was then washed with $1 \mathrm{M}$ $\mathrm{HCl}$ in deionized water to remove the remaining salts and continuously washed with the deionized water until a neutral $\mathrm{pH}$ was achieved. The obtained sample denoted activated carbon (AC), was then oven-dried at $80{ }^{\circ} \mathrm{C}$ for $24 \mathrm{~h}$ followed by characterization using various techniques. 


\subsection{Physico-chemical characterization}

X-ray photoelectron spectroscopy (XPS) technique was used to identify the surface moieties and quantify the amount in percentage present in the activated carbon in pellet form. The pellets were made with the aid of the Polytetrafluoroethylene (PTFE) binder. A Physical Kratos instrument AXIS SUPRA x-ray photoelectron spectroscopy (XPS) equipment at University of South Africa (UNISA) was used for the analysis. The spectra were obtained at pass energy of $117.5 \mathrm{eV}$, to acquire the elemental configuration of the sample. Deconvolution of core-level C1s and O1s sections were captured at pass energy of $23.5 \mathrm{eV}$, incremental step of $0.05 \mathrm{eV}$. Raman spectroscopy analyses were performed with a 300R+ confocal WiTec instrument (WiTec $\mathrm{GmbH}$ ), while the gas sorption analyses was performed with a Micromeritics TriStar II 3020 (version 2.00) analyzer at $-196{ }^{\circ} \mathrm{C}$. The surface area was obtained by the Brunauer-Emmett-Teller (BET) technique from the adsorption isotherm in the relative pressure range $(\mathrm{P} / \mathrm{P} 0)$ of $0.01-1$. Scanning electron micrographs were acquired using a Zeiss Ultra Plus 55 field emission scanning electron microscope (FE-SEM) working at a voltage of $2.0 \mathrm{kV}$.

\subsection{Electrochemical measurements}

The electrochemical measurements was performed on a VMP300 Bio-Logic instrument, France) at room temperature. The electrodes were made by integrating the active material with carbon black and polyvinylidene fluoride (PVDF) binder in the following weight ratio 80: 10: 10 in N-Methyl-2-pyrrolidone (NMP) to make a paste was then homogeneously coated on a carbon paper current collector and dried at $60{ }^{\circ} \mathrm{C}$ in an electric oven overnight. The tests were performed in a two-electrode symmetric supercapacitors fabricated using the AC as anode and cathode materials, with each electrode having a mass of $\sim 1.5 \mathrm{mg}$ separated by 
a $0.18 \mu \mathrm{m}$ thick microfiber glass filter paper in coin cell with $6 \mathrm{M} \mathrm{KOH}$ alkaline electrolyte. The cyclic voltammetry (CV) and constant current galvanostatic charge-discharge (CGCD) measurement for the device was investigated at different scan rates and current densities. Electrochemical impedance spectroscopy (EIS) was measured in a frequency span of $100 \mathrm{kHz}$ to $0.01 \mathrm{~Hz}$.

The total capacitance (F) of a symmetric supercapacitor device is a reflection of the charges stored at a specific voltage and is calculated from the slope of the CGCD

$C_{T}=\frac{I \Delta t}{\Delta V}$

The specific capacitance $\left(\mathrm{F} \mathrm{g}^{-1}\right)$ for the single electrode was obtained using $[26,27]$ :

$C_{s}=\frac{4 C_{T}}{m}$

where $\mathrm{I}$ is the current (A), $\Delta \mathrm{t}$ is the discharge or charge duration(s), $\Delta V=V_{o}-V_{I R-d r o p}$ is the change in cell voltage cell without the IR drop and $m$ is the mass of carbon in each of the two electrodes (g).

The resistance $(\Omega)$ was calculated from the difference of the voltage at the initial stage of the discharge from the CCCD curves at all the current densities using equation 3 [27].

$R=\frac{\Delta V}{2 I}$

The stability test of the PPY derived carbon was carried out based on floating as shown in scheme 1 and the capacitance was calculated after the IR drop has been subtracted [23]. The floating test is described as follows: After every $10 \mathrm{~h}$ of aging five CGCD sequence at the maximum voltage $\left(\mathrm{V}_{\mathrm{H}}\right)$ was followed and the specific capacitance was calculated from the 
first and fifth discharge respectively. These sequences were reiterated 20 times, i.e. a total floating time of $200 \mathrm{~h}$ [28]. The resistance (ESR) which is a measure of the conductivity of the material was also estimated from the IR drop at the initial step discharge curve as shown in scheme 1.

\section{RESULTS}

An XPS analysis was carried out to establish the surface functionalities and quantify the chemical composition of the AC sample made into pellet with the aid of a Polytetrafluoroethylene (PTFE) binder. Fig. 1 (a) shows the XPS spectra for of AC pellet and binder in the wide-scan range exhibiting signals arising from carbon and fluorine bonds from the PTFE pellet and carbon and oxygen atoms for the APC pellet. Fig. 1 (b) shows the C1s core-level spectra of the samples which provide more insight to the chemical structures of the sample. The peaks at binding energy of $290.9 \mathrm{eV}$ and $294.4 \mathrm{eV}$ are assigned to the $\mathrm{CF}_{2}$ and $\mathrm{CH}_{2}$ groups of PTFE, respectively. The $\mathrm{C} 1 \mathrm{~s}$ core level peak of the AC pellet consists of peaks observed in the PTFE binder with three additional peaks observed in the C1s corelevel of the AC sample and can be fitted into three contributions at $284.6 \mathrm{eV}(-\mathrm{COOH}), 285.2$ $\mathrm{eV}(\mathrm{C}=\mathrm{C})$, and $289.0 \mathrm{eV}(\mathrm{C}=\mathrm{O})$. This signifies the presence of graphitic carbon and functional carbon in the form of carboxyl or ether groups. The $01 \mathrm{~s}$ peaks at 532.0 and $533.2 \mathrm{eV}$ (Figure 1 (c)) can be ascribed to oxygen in the form of (O-C) and (C-O), respectively $[29,30]$. 

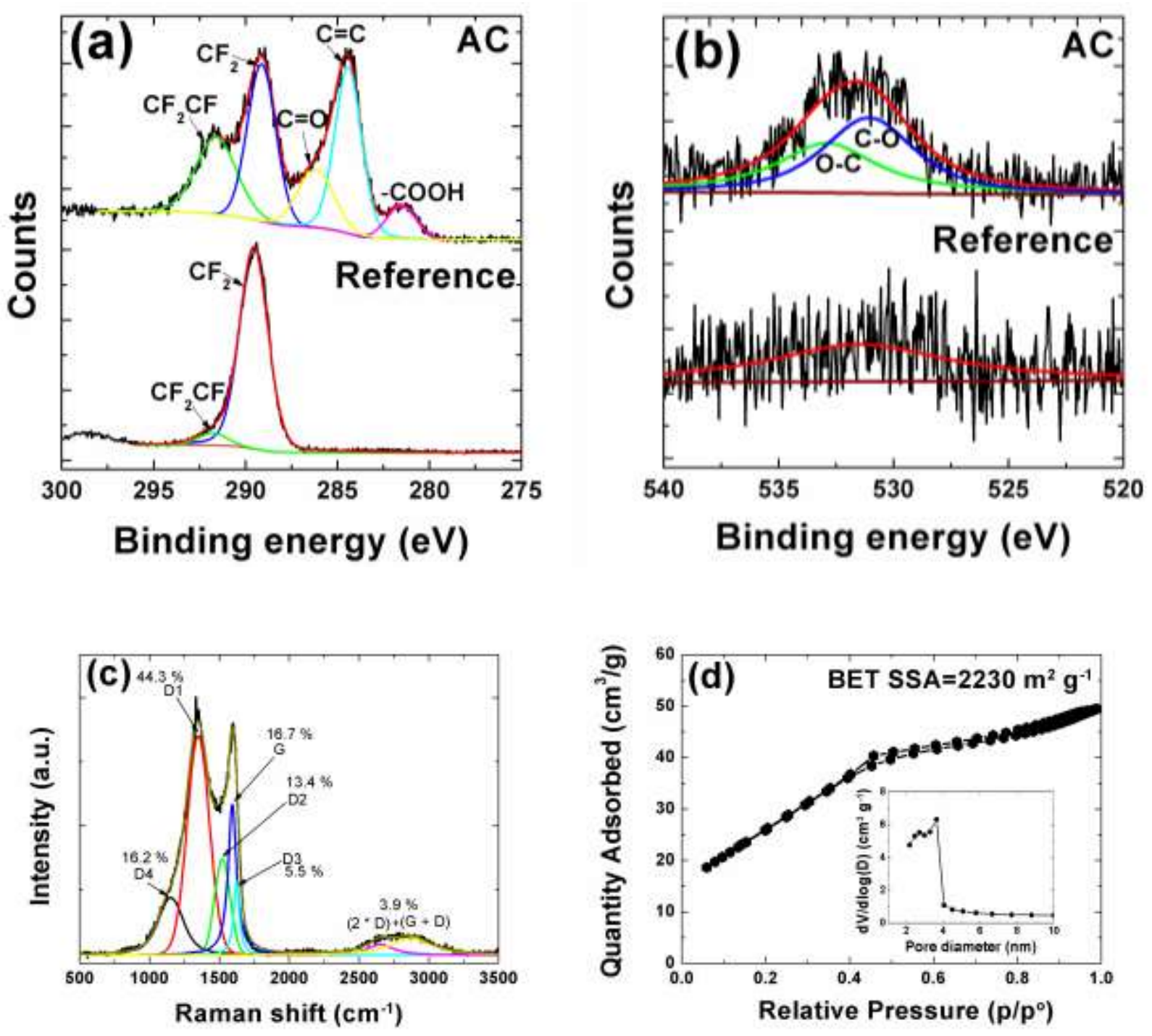

Figure 1 XPS curves of (a) wide scan, (b) C 1s, and (c) O 1s regions for AC material and the PTFE reference binder.

Raman spectroscopy remained one of the significant procedure for the analysis of textural defects in graphitic materials [31]. Fig. 2 presents Raman spectrum of the activated carbon with obvious peaks at $\sim 1354 \mathrm{~cm}^{-1}, \sim 1598 \mathrm{~cm}^{-1}$ and a broad peak between $\sim 2700 \mathrm{~cm}^{-1}$ and $2900 \mathrm{~cm}^{-1}$ assigned to the $\mathrm{D}, \mathrm{G}$ and 2D-bands of carbon material, respectively represented by black average spectrum. The peaks were deconvoluted to analyse different vibrational modes of the produced carbon material in details with a Lorentzian curve fittings of the different combinations in accordance with reported literature for other carbon materials 


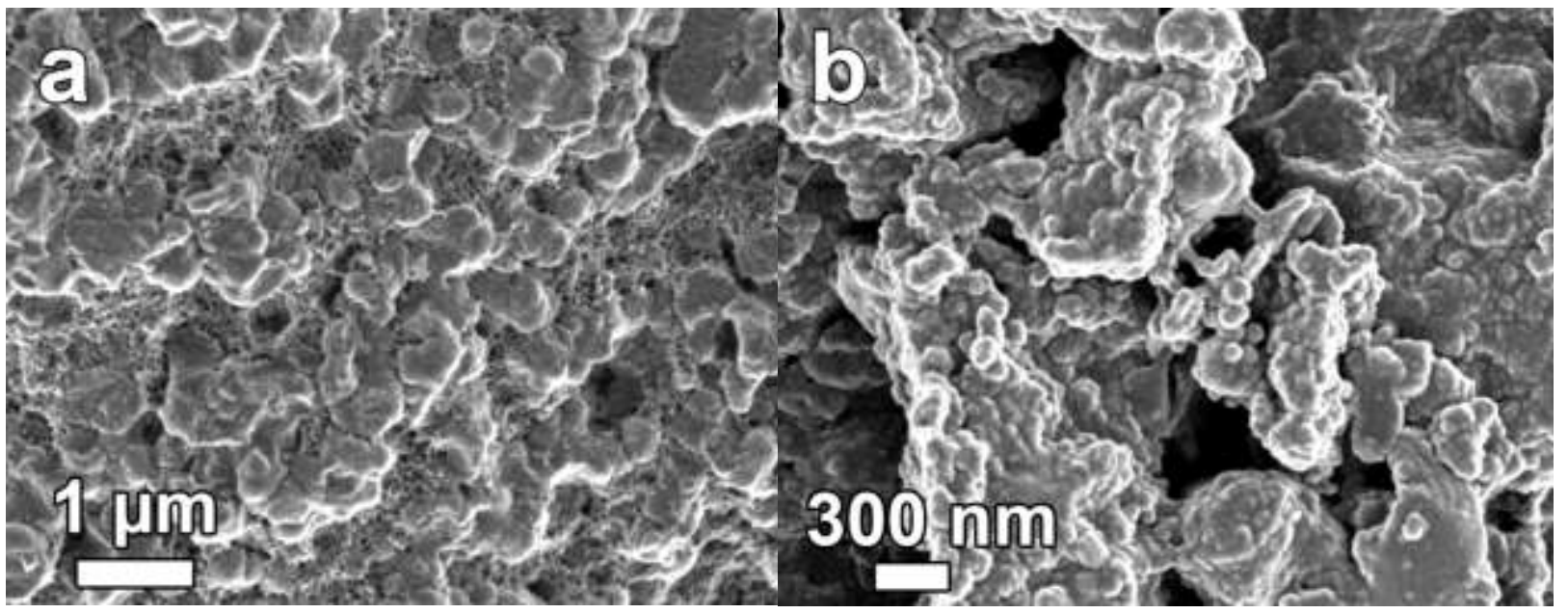

Figure 2 Raman spectra $\left(\lambda_{0}=514 \mathrm{~nm}\right)$ of the activated carbon and the corresponding fitting of different

combinations of the Raman peaks and the percentages contribution of each peak.

(e.g. soot in Ref. [32]): The $\mathrm{G}$ band arises from the in-plane vibration stretching of the $\mathrm{C}-\mathrm{C}$ bond in graphitic materials and is common to all $\mathrm{sp}^{2}$ carbon materials [33]. The D-peak, is due to the breathing modes of $\mathrm{sp}^{2}$ rings activated through a dual resonance effect in the presence of defects [34]. The 2D band is the second order mode of the D-peak. The high intensity of the D-band in the spectrum is attributed to the presence of a sizeable amount of defects in the activated carbon. The deconvoluted bands are described as follows; D1 arises from the edge of a probable graphene-sheet carbon atoms and the edge planes perpendicular to the graphene sheets of the bulk carbon materials [35]. The origin of D2 is related to a lattice vibration equivalent to that of the $\mathrm{G}$ band but involving surface graphene sheets which are not directly intercalated between graphene sheets in the bulk of a carbon material [32]. The D3 band arises from the circulation of amorphous carbon in interstitial places in the lattice of the carbon material [36]. The D4 band is as a result of lattice vibrations corresponding to $\mathrm{sp}^{2}-\mathrm{sp}^{3}$ bonds $[37,38]$. Lastly, the two peaks at $\sim 2700 \mathrm{~cm}^{-1}$ and $2900 \mathrm{~cm}^{-1}$ have been assigned to the (2D) overtone and (G+D) combination, respectively [32]. The intensity of the G-band to the intensity of the D-band $I_{D} / I_{G}$ for the sample was 1.0, indicating a low degree of graphitic crystalline structure. It is worth stating that only 
the $G$ peak is susceptible to material conditions such as temperature, doping, and significant amounts of strain present in the material [33,34,39]. From the analyses above it is reasonable to conclude that the sample contained a little degree of graphitization with its structural unit mostly consists of defected carbon.

The surface texture of the activated carbon was investigated by $\mathrm{N}_{2}$ gas adsorption and desorption analysis via the BET method. The BET reveals a high SSA value of $2230 \mathrm{~m}^{2} \mathrm{~g}^{-1}$ shown in Fig. 3 (a) with average pore volume of $1.86 \mathrm{~cm}^{3} \mathrm{~g}^{-1}$. The Figure reveals type VI isotherm featuring hysteresis loop generated by the capillary condensation of the adsorbate in the micro- and mesopores of the carbon material. It is worth noting that both pores are important for charge storage; with micropores responsible for ion traps for energy storage

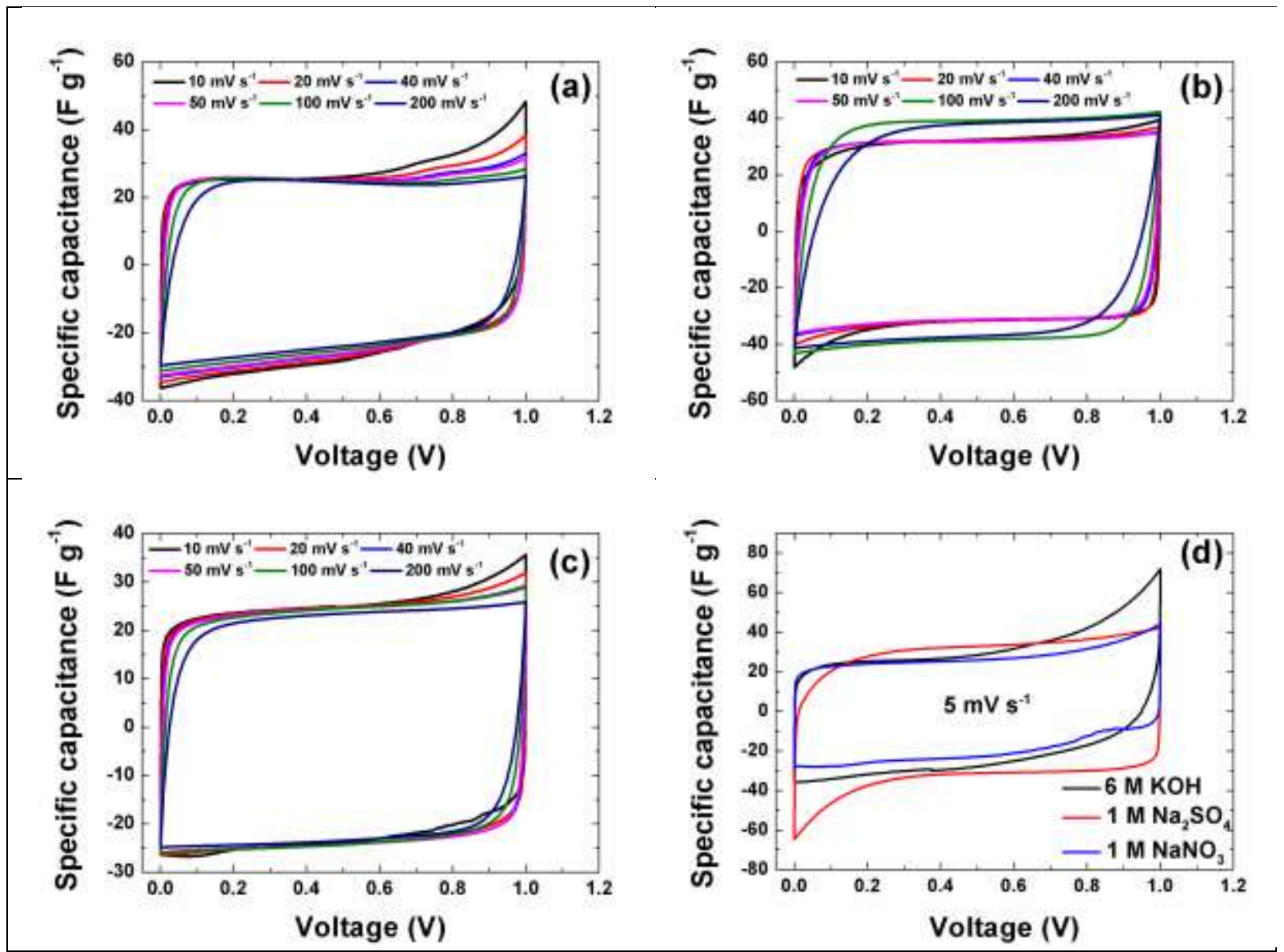

Figure 3 (a) $\mathrm{N}_{2}$ isotherm (adsorption/desorption) (b) pore size distribution of the AC material. 
and the mesopores act as channels for ion transport from the electrolyte to the interface between the electrode and electrolyte [18]. The Barrett Joyner Halenda (BJH) pore size distribution (PSD) is presented in Fig. 3 (b) primarily showing mesopores; the presence of micropore is established by the behavior of the PSD curve $\sim 2 \mathrm{~nm}$. It is worth noting that the BET system used in this work could not go to a lower pressure which is necessary to see the pore size of the micropore.

SEM micrographs gives a good Insight and information into the structure and morphology of AC material. The SEM micrographs in Fig. 4 (a) (low magnification) and (b) (high magnification) show that the AC comprise exclusively large amount of homogeneous random structures with larger grains in the range of $70-100 \mathrm{~nm}$ that are embedded into
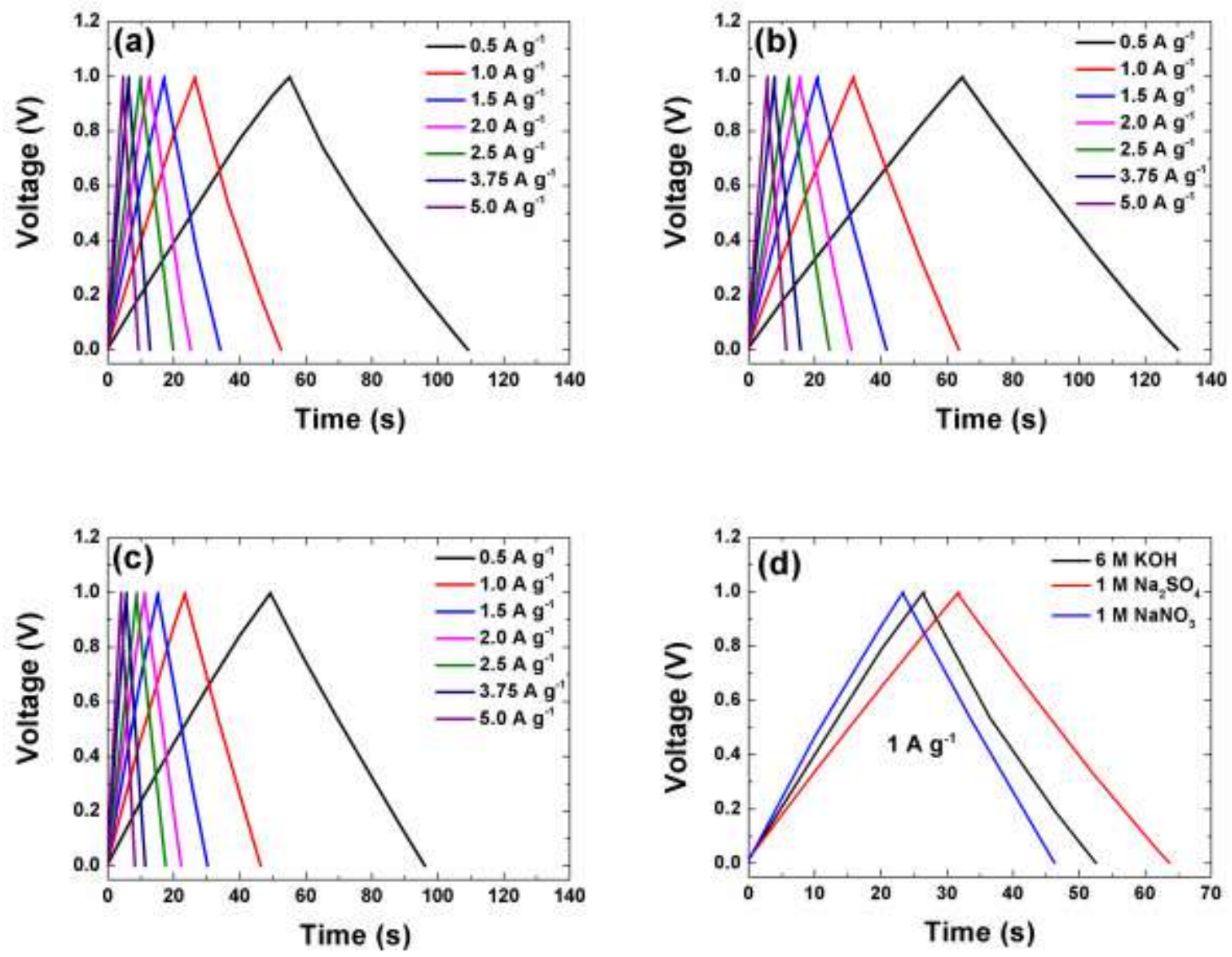

Figure 4 SEM micrographs (a) low and (b) high magnification of the AC respectively 
agglomerated and interconnected nanostructures. Generally, alkaline $\mathrm{KOH}$ activation of materials leads to porous structures on the surface of the material, in our case the activation and carbonization process leads to creation of micro-, meso- pores along with the macro- pores and agglomerated particles inherited from the PPY posing a 3D structure with a very high BET surface area as discussed previously which could be favorable for electrochemical applications.

To evaluate the performance of the electrode material, a symmetric supercapacitor device was fabricated and optimized for high performance at an optimum allowable operating voltage of $1.2 \mathrm{~V}$ due to the thermodynamical decomposition of water at $1.23 \mathrm{~V}$. The cyclic voltammograms (CVs) of a symmetric cell with a slow sweep rates between $5-50 \mathrm{mV} \mathrm{s}^{-1}$ is shown in Fig. 5 (a). It is observed that the device exhibited a double layer mechanism without noticeable faradaic contributions suggesting that the evolution of oxygen or dihydrogen of the aqueous (water) electrolyte was not reached within this voltage window showing good thermodynamic stability of the electrodes materials in the electrolyte. Fig. 5
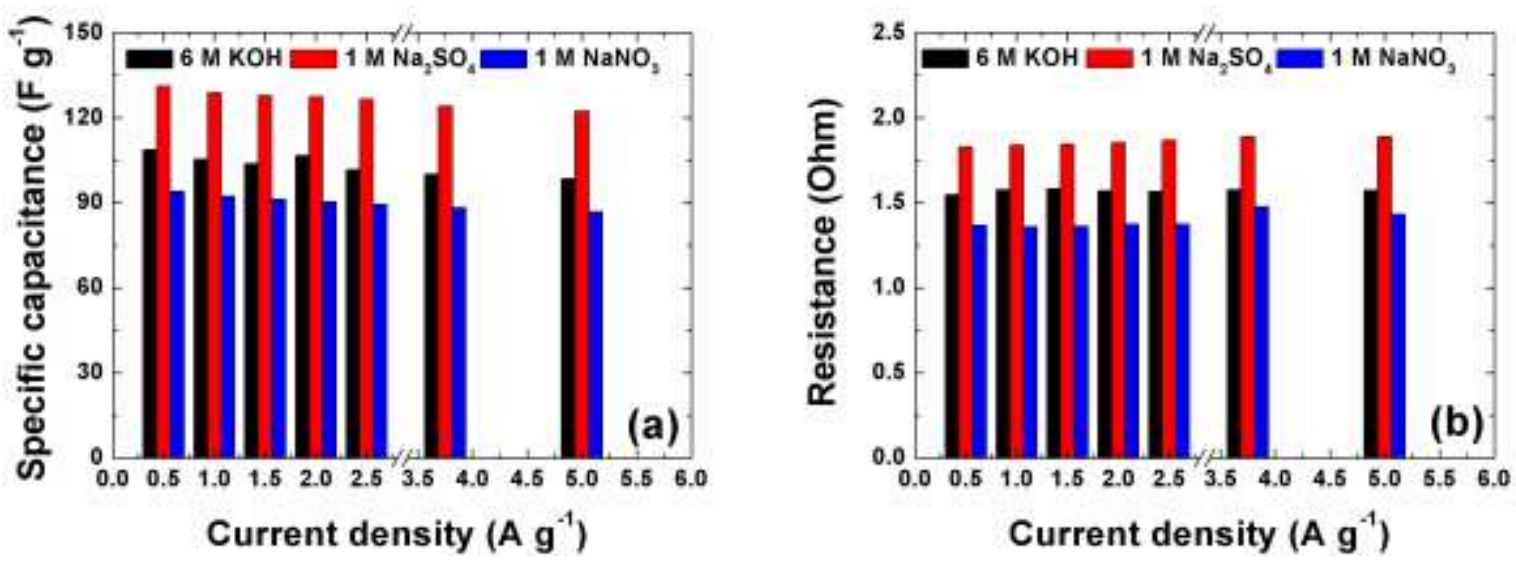

Figure 5 (a-b) CV of symmetric cell of PPY derived carbon at different scan rate, (c) charge-discharge curve at different current density from $0.5 \mathrm{~A} \mathrm{~g}^{-1}-10 \mathrm{~A} \mathrm{~g}^{-1}$, (d) specific capacitance and (e) the resistance change with the current density 
(b) shows CV curves from $100 \mathrm{mV} \mathrm{s}^{-1}$ to $1 \mathrm{~V} \mathrm{~s}^{-1}$. Remarkably, the $\mathrm{CV}$ shapes are still rectangular in shape. At $1 \mathrm{~V} \mathrm{~s}^{-1}$ the $\mathrm{CV}$ still maintains almost rectangular behavior signifying excellent rate capability of the AC electrode material. The characteristic CGCD curves at different current densities are shown in Fig. 5 (c). The curves displays almost perfect symmetric triangular feature, indicating an EDL capacitor behavior meaning that the cell possesses excellent electrochemical reversibility with very small Ohmic drop, affirming the results from the $\mathrm{CV}$ measurements. The resultant specific capacitance estimated from the discharge curves in Fig. 5 (d) presents a specific capacitance of $179 \mathrm{~F} \mathrm{~g}^{-1}$ at a current density of $0.5 \mathrm{~A} \mathrm{~g}^{-1}$ and $158 \mathrm{~F} \mathrm{~g}^{-1}$ at a current density of $10 \mathrm{~A} \mathrm{~g}^{-1}$ representing a capacitance dropped of $\sim 11.7 \%$ attributed to a fast ion movement at reasonably high current density. These specific capacitance results obtained are influenced by the presence of oxygenated surface functional that modify the electrostatic field on the surface, and could also increase the wettability of electrode thus making the accessibility of more micropores easy during the electrochemical process.

The dependence of the Ohmic resistance on increasing current density is presented in Fig. 5 (e). The Figure Shows the resistance calculated from the IR drop of the discharge curve using equation 3 as a function of the current density. A constant resistance as the current increases is observed which attests the non-existence of faradaic reactions as observed from the $\mathrm{CV}$ and $\mathrm{CD}$ measurements.

As stated earlier, the voltage holding or floating test (accelerated ageing) has emerged as a reliable technique to evaluate the stability ECs rather than the convention charge-discharge over several thousand of cycles. Thus, the symmetric cell was subjected to floating at $1.2 \mathrm{~V}$ at $5 \mathrm{~A} \mathrm{~g}^{-1}$ in order to determine the stability of the electrodes on the cycle life and how long 
it takes before the electrodes begin to degrade. A $10 \mathrm{~h}$ voltage holding period is trailed by five CCCD cycles to detect any loss in the cell capacitance over $190 \mathrm{~h}$. Fig. 6 (a) displays, the regression of cell capacitance with floating over a time of $190 \mathrm{~h}$. From the Figure, it is evident that the cell voltage is stable within this voltage window and time with a capacitance loss of $\sim 25 \%$. Fig. 6 (b) presents the resistance as a function of the floating time, from this Figure; it is observed that the resistance was constant up to $~ 120 \mathrm{~h}$ of voltage holding. After $120 \mathrm{~h}$ of floating, the resistance increases rapidly to $\sim 4.5 \Omega$ at the end of 190 $\mathrm{h}$ indicating capacitance loss and gradual degradation of the electrode possibly attributed to higher potential attained by the positive electrode [40]. This shows that the capacitance decay at $120 \mathrm{~h}$ of holding time was $\sim 9 \%$ and rapidly decreased afterwards due to the increase in resistance. The capacitance drop and increasing resistance are most likely due to reduced ion accessibility (electrolyte starvation effect) or to the reduced accessible electrode surface area for ions [41]. However, taking into account the widely acceptable criteria to measure end-of-life for supercapacitor ( $20 \%$ or $30 \%$ capacitance decay) $[24,42]$, it is reasonable to conclude that the electrode materials investigated are good and are within the acceptable values as potential electrode for electrochemical applications.
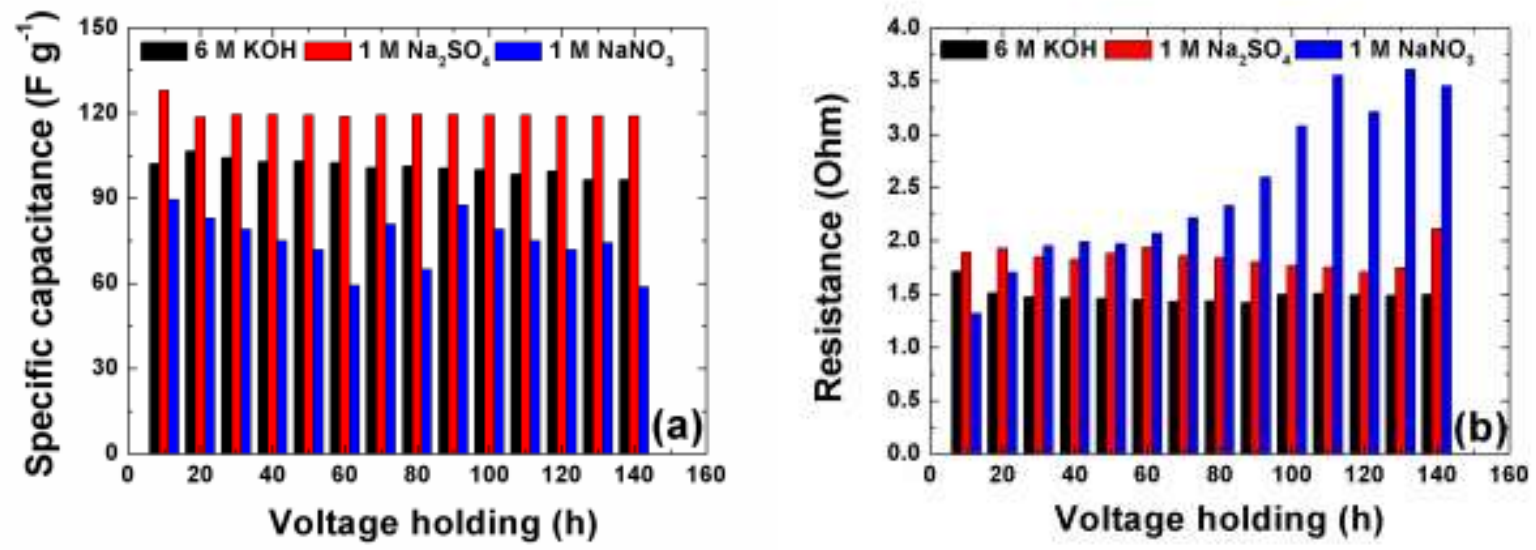

Figure 6 (a) the specific capacitance and (b) the resistance as a function of the floating time. 
Electrochemical impedance spectroscopy (EIS) is fundamental to understand the behavior of the electrodes at open circuit potential or at a particular AC signal. The Nyquist plot is presented in Fig. 7 (a) exhibiting a semicircle in the high to mid-frequency region representing the charge transfer resistance $\left(R_{C T}\right)$, and a vertical line in the low frequency region, indicative of an ideal capacitive behavior of the $A C$ electrode material. The intercept on the real $Z^{\prime}$ axis of Nyquist (inset) is the solution resistance $\left(R_{S}\right)$. The $R_{S}$ generally originates from the total resistance of electrolyte, the resistance of the active materials and the
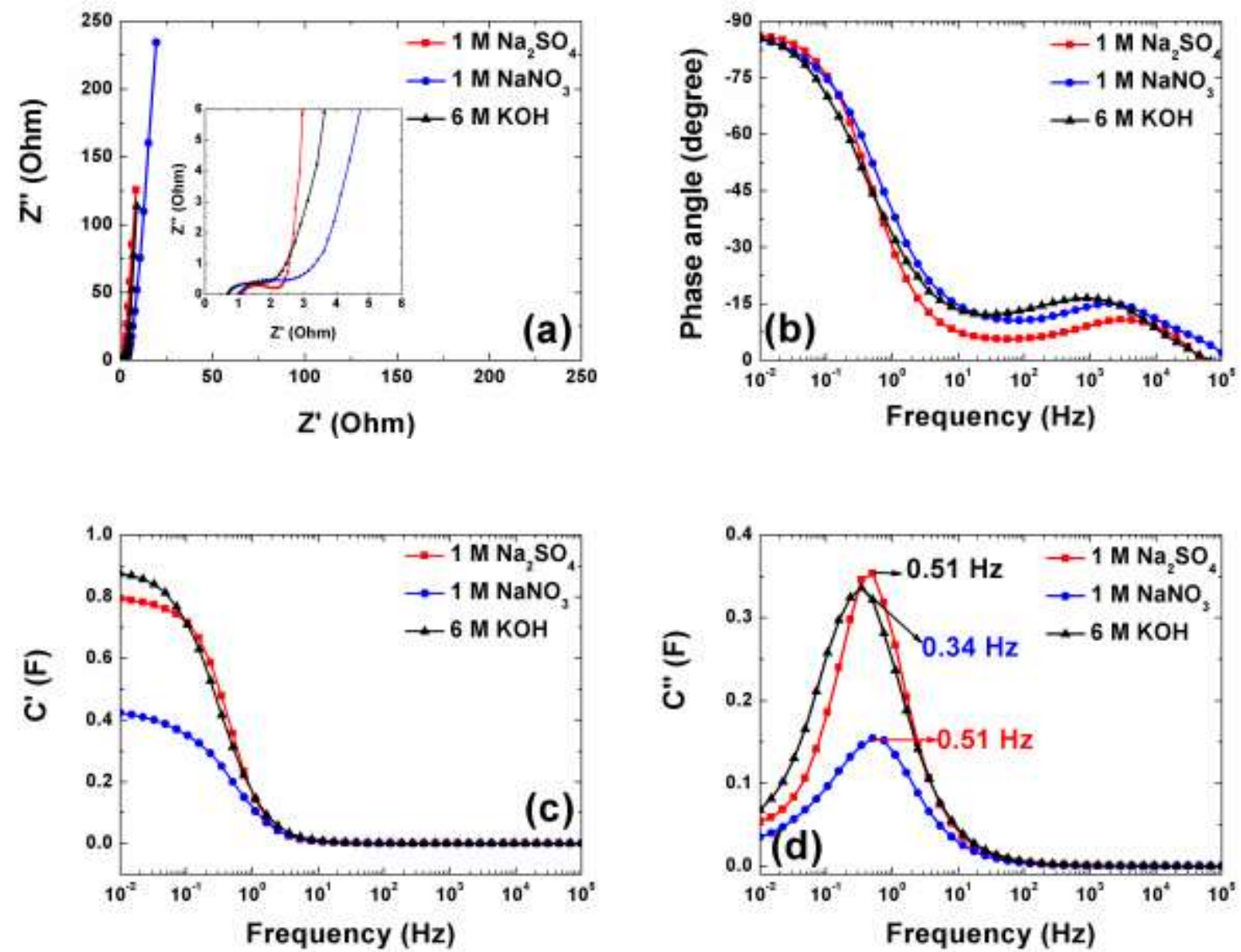

Figure 7 (a) EIS Nyquist plot with the fitting and equivalent circuit, (b) the CV before and after floating (c) Nyquist before and after floating, (d) C' and C" as function of frequency and (e) Bode plot of the symmetric cell before and after. 
resistance at the interface between the active electrode material and current collector [43]. From the figure the $R_{S}$ and $R_{C T}$ of the symmetric device are $0.65 \Omega$ and $0.17 \Omega$ respectively. The equivalent circuit and fitting of the EIS shown as inset to the figure was evaluated with a ZFIT/EC-Lab software.

The circuit consists of $R_{s}$ in series with capacitance $(C)$, and the $C$ is placed in parallel with the $R_{C T}$, which is connected in series with the constant phase element (Q). $Q$ is placed in parallel with the Warburg (W) and the leakage resistance $\left(R_{L}\right)$ which are in series. The Warburg diffusion element region governs the ions diffusion within porous electrode materials. At very low frequencies, a polarizable electrode gives rise to a line parallel to the vertical axis; from Fig. 7 (a) there is a variation from this ideal behavior. The variation is due to the existence of a resistive quantity linked with $\mathrm{Q}$. This resistance quantity is known as leakage resistance symbolized by $R_{L}$ in circuit diagram inset to Fig. 7 (a). The parameters obtained from the fitting of the Nyquist plot is shown in Table 1 below. The $R_{S}$ and $R_{C T}$ values and other parameters are in good agreement with experimental data.

Table 1. Fitting parameters for the equivalent circuit.

$R_{S}(\Omega) Q\left(F . s^{(a-1)}\right) R_{L}(\Omega) R_{C T}(\Omega) C(\Omega)$

$\begin{array}{lllll}0.65 & 0.067 & 4 & 0.176 & 0.4\end{array}$

As a proof of the stability and performance of the electrode material CV and EIS were measured after voltage holding for $200 \mathrm{~h}$. Fig. 7 (b) presents the CV $\left(50 \mathrm{mV} \cdot \mathrm{s}^{-1}\right)$ of the cell before and after floating test, revealing a nearly rectangular shape up to $1.2 \mathrm{~V}$ suggesting a small loss in capacitance as discussed in Fig. 6 (a) above. The comparative CV's clearly shows that voltage holding or floating test (aging) does not have a significant degradation effect on the electrodes of the symmetric device. The Nyquist after voltage holding is presented in 
Fig. 7 (c), showing that there was no much deviation to the initial curve confirming that very small degradation of the electrode material transpired. The Nyquist plots before and after floating exhibit comparable internal resistance values $\left(E S R\left(R_{S}\right)=0.65 \Omega\right.$ and $0.98 \Omega$ respectively) shown in the inset. The charge transfer resistance $R_{C T}$ value of $0.17 \Omega$ (semicircle region) remains unchanged, denoting a good contact between activated carbon and current collectors.

Time constant is another parameter for evaluation of the responsiveness of a supercapacitor device. The time constant is defined by $\tau=R \times C$ where $\mathrm{R}$ is the equivalent series resistance and $\mathrm{C}$ is the total device capacitance at the lowest frequency. Based on the above relation a time constant of $73.6 \mathrm{~ms}$ was calculated demonstrating a quick response of the electrodes which could be suitable for applications such as AC filtering at desirable frequency. The time constant $(\tau)$ was also compared with the relaxation time constant $\left(\tau_{0}\right)$. The $\tau_{0}$ is usually obtained from the EIS by plotting the real and imaginary capacitance of the cell with the change in the frequency as shown in Fig. 7 (d). $C^{\prime}$ represents the deliverable capacitance at the lowest frequency $(0.46 \mathrm{~F})$, and the $\mathrm{C}^{\prime \prime}$ explains the changeover frequency between a pure capacitive and a pure resistive behavior which symbolises the energy loss because to the irreversible process of the electrodes [26]. $\tau_{0}=1 / f_{0}$ at the position where $C^{\prime \prime}$ reaches a maximum at frequency $f_{0}(2.45 \mathrm{~Hz})$ defining a relaxation time of $0.4 \mathrm{~s}$. This value implies that the $50 \%$ of the cell capacitance can be reached within a short time of $0.4 \mathrm{~s}$ at low frequency. The change in frequency with the phase angle before and after floating test is presented in Fig. 7 (e). The phase angle for the electrode show no significant change showing similar values $\sim-87.5^{\circ}$ and $\sim-87.5^{\circ}$, very near to $-90^{\circ}$, thus describing a perfect capacitive response. 


\section{CONCLUSION}

Activated carbon (AC) was prepared by carbonising a conducting polymer PPY. The texture and mesoporous architecture of the AC reveals a relatively SSA of $2230 \mathrm{~m}^{2} \mathrm{~g}^{-1}$ with a micropore volume of $1.86 \mathrm{~cm}^{3} \mathrm{~g}^{-1}$ which could be beneficial for energy storage. The performance of a symmetric capacitors in $6 \mathrm{M} \mathrm{KOH}$ a electrolyte was demonstrated showing a specific capacitance of $179 \mathrm{~F} \mathrm{~g}^{-1}$ at $0.5 \mathrm{~A} \mathrm{~g}^{-1}$ and $158 \mathrm{~F} \mathrm{~g}^{-1}$ at $10 \mathrm{~A} \mathrm{~g}^{-1}$ suggesting a high rate capability of the symmetric EDLC device. Voltage holding or floating was used to evaluate the stability of the symmetric cell at $1.2 \mathrm{~V}$ for $190 \mathrm{~h}$ rather than the conventional cycling test because floating of AC porous electrodes for several hours is susceptible to degradation and subsequently deterioration of the performance of the device. However, at the end of the floating for $190 \mathrm{~h} \sim 25 \%$ decay in capacitance (i.e. from $160.5 \mathrm{~F} \mathrm{~g}^{-1}$ to $120 \mathrm{~F} \mathrm{~g}^{-1}$ at $5 \mathrm{~A} \mathrm{~g}^{-1}$ ) of the symmetric device was observed with increase in resistance which represent a decent and interesting performance of the symmetric device. Largely, the origin of the capacitance fading was ascribed to electrolyte starvation effect) or to the reduced accessible electrode surface area for charge storage. Nevertheless, considering the widely acceptable criteria to measure end-of-life for supercapacitor ( $20 \%$ or $30 \%$ capacitance decay), these results suggest that the PPY derived carbon based material has a huge possibility in high performance and continuous application.

\section{ACKNOWLEDGEMENT}

This work is based on the research supported by the South African Research Chairs Initiative of the Department of Science and Technology and National Research Foundation of South Africa (Grant No. 97994). Any opinion, finding and conclusion or recommendation expressed in this material is that of the author(s) and the NRF does not accept any liability in this 
regard. A. Bello acknowledges the University of Pretoria's financial support for their Postdoctoral fellowship.

\section{REFERENCES}

[1] J. Yan, Q. Wang, T. Wei, Z.J. Fan, Recent Advances in Design and Fabrication of Electrochemical Supercapacitors with High Energy Densities, Adv. Energy Mater. 4 (2014) 43.

[2] P. Simon, Y. Gogotsi, Capacitive energy storage in nanostructured carbon--electrolyte systems, Acc. Chem. Res. 46 (2012) 1094-1103.

[3] B.E. Conway, Electrochemical Supercapacitors: Scientific Fundamentals and Technological Applications, Kluwer Academic/Plenum: New York, 1999.

[4] P. Simon, Y. Gogotsi, Materials for electrochemical capacitors, Nat. Mater. 7 (2008) 845-854.

[5] Y. Korenblit, M. Rose, E. Kockrick, L. Borchardt, A. Kvit, S. Kaskel, G. Yushin, High-rate electrochemical capacitors based on ordered mesoporous silicon carbide-derived carbon, ACS Nano. 4 (2010) 1337-1344.

[6] V. Presser, M. Heon, Y. Gogotsi, Carbide-Derived Carbons-From Porous Networks to Nanotubes and Graphene, Adv. Funct. Mater. 21 (2011) 810-833.

[7] B.G. Choi, M. Yang, W.H. Hong, J.W. Choi, Y.S. Huh, 3D macroporous graphene frameworks for supercapacitors with high energy and power densities, ACS Nano. 6 (2012) 4020-4028.

[8] L.L. Zhang, R. Zhou, X.S. Zhao, Graphene-based materials as supercapacitor electrodes, J. Mater. Chem. 20 (2010) 5983-5992.

[9] Y. Gao, Y.S. Zhou, M. Qian, X.N. He, J. Redepenning, P. Goodman, H. M. Li, L. Jiang, Y. F. Lu, Chemical activation of carbon nano-onions for high-rate supercapacitor electrodes, Carbon 
$51(2013)$ 52-58.

[10] M. Kubota, A. Hata, H. Matsuda, Preparation of activated carbon from phenolic resin by $\mathrm{KOH}$ chemical activation under microwave heating, Carbon 47 (2009) 2805-2811.

[11] S. Dörfler, I. Felhösi, T. Marek, S. Thieme, H. Althues, L. Nyikos, S. Kaskel, High power supercap electrodes based on vertical aligned carbon nanotubes on aluminum, J. Power Sources. 227 (2013) 218-228.

[12] E. lyyamperumal, S. Wang, L. Dai, Vertically aligned BCN nanotubes with high capacitance, ACS Nano. 6 (2012) 5259-5265.

[13] F.B. E. Frackowiak, E. Frackowiak, F. Beguin, F. Béguin, Carbon materials for the electrochemical storage of energy in capacitors, Carbon 39 (2001) 937-950.

[14] F. Béguin, V. Presser, A. Balducci, E. Frackowiak, Carbons and electrolytes for advanced supercapacitors., Adv. Mater. 26 (2014) 2219-51, 2283.

[15] P. Hapiot, C. Lagrost, Electrochemical reactivity in room-temperature ionic liquids, Chem. Rev. 108 (2008) 2238-2264.

[16] A. Bello, F. Barzegar, D. Momodu, J. Dangbegnon, F. Taghizadeh, N. Manyala, Symmetric supercapacitors based on porous 3D interconnected carbon framework, Electrochim. Acta. 151 (2015) 386-392.

[17] L. Demarconnay, E. Raymundo-Piñero, F. Béguin, A symmetric carbon/carbon supercapacitor operating at 1.6V by using a neutral aqueous solution, Electrochem. Commun. 12 (2010) $1275-1278$.

[18] K. Fic, G. Lota, M. Meller, E. Frackowiak, Novel insight into neutral medium as electrolyte for high-voltage supercapacitors, Energy Environ. Sci. 5 (2012) 5842-5850. 
[19] B. Qiu, C. Pan, W. Qian, Y. Peng, L. Qiu, F. Yan, Nitrogen-doped mesoporous carbons originated from ionic liquids as electrode materials for supercapacitors, J. Mater. Chem. A. 1 (2013) 6373.

[20] Y. Xu, J. Wang, Z. Chang, B. Ding, Y. Wang, L. Shen, C. Mi, H. Dou, X. Zhang, Facile Synthesis of Nitrogen-Containing Mesoporous Carbon for High-Performance Energy Storage Applications, Chem. A Eur. J. 22 (2016) 4256-4262.

[21] F. Barzegar, A. Bello, O.O. Fashedemi, J.K. Dangbegnon, D.Y. Momodu, F. Taghizadeh, N. Manyala, Synthesis of 3D porous carbon based on cheap polymers and graphene foam for high-performance electrochemical capacitors, Electrochim. Acta. 180 (2015) 442-450.

[22] V. Ruiz, R. Santamar'ia, M. Granda, C. Blanco, Long-term cycling of carbon-based supercapacitors in aqueous media, Electrochim. Acta. 54 (2009) 4481-4486.

[23] D. Weingarth, H. Noh, A. Foelske-Schmitz, A. Wokaun, R. Kötz, A reliable determination method of stability limits for electrochemical double layer capacitors, Electrochim. Acta. 103 (2013) 119-124.

[24] D. Weingarth, A. Foelske-Schmitz, R. Kötz, Cycle versus voltage hold - Which is the better stability test for electrochemical double layer capacitors?, J. Power Sources. 225 (2013) 8488.

[25] Y. Zhu, S. Murali, M.D.M. Stoller, K.J. Ganesh, W. Cai, P.J. Ferreira, A. Pirkle, R. M. Wallace, K. A. Cychosz, M. Thommes, D. Su, E. A. Stach, R. S. Ruoff, Carbon-based supercapacitors produced by activation of graphene, Science. 332 (2011) 1537-41.

[26] P.L. Taberna, P. Simon, J.-F.F. Fauvarque, Electrochemical characteristics and impedance spectroscopy studies of carbon-carbon supercapacitors, J. Electrochem. Soc. 150 (2003) A292-A300. 
[27] S. Zhang, N. Pan, Supercapacitors Performance Evaluation, Adv. Energy Mater. 5 (2015) 1401401.

[28] P.W. Ruch, D. Cericola, A. Foelske-Schmitz, R. Kötz, A. Wokaun, Aging of electrochemical double layer capacitors with acetonitrile-based electrolyte at elevated voltages, Electrochim. Acta. 55 (2010) 4412-4420.

[29] H. Li, Z. Kang, Y. Liu, S.-T. Lee, Carbon nanodots: synthesis, properties and applications, J. Mater. Chem. 22 (2012) 24230-24253.

[30] Q. Wang, X. Liu, L. Zhang, Y. Lv, Microwave-assisted synthesis of carbon nanodots through an eggshell membrane and their fluorescent application, Analyst. 137 (2012) 5392-5397.

[31] M.A. Pimenta, G. Dresselhaus, M.S. Dresselhaus, L.G. Cancado, A. Jorio, R. Saito, Studying disorder in graphite-based systems by Raman spectroscopy, Phys. Chem. Chem. Phys. 9 (2007) 1276-1290.

[32] A. Sadezky, H. Muckenhuber, H. Grothe, R. Niessner, U. Pöschl, Raman microspectroscopy of soot and related carbonaceous materials: Spectral analysis and structural information, Carbon 43 (2005) 1731-1742.

[33] L.M.M. Malard, M. a. A. Pimenta, G. Dresselhaus, M.S.S. Dresselhaus, Raman spectroscopy in graphene, Phys. Rep. 473 (2009) 51-87.

[34] A.C. Ferrari, J.C. Meyer, V. Scardaci, C. Casiraghi, M. Lazzeri, F. Mauri, S. Piscanec, D. Jiang, K. S. Novoselov, S. Roth, A. K. Geim, Raman Spectrum of Graphene and Graphene Layers, Phys. Rev. Lett. 97 (2006) 187401.

[35] Y. Wang, D.C. Alsmeyer, R.L. McCreery, Raman spectroscopy of carbon materials: structural basis of observed spectra, Chem. Mater. 2 (1990) 557-563.

[36] T. Jawhari, A. Roid, J. Casado, Raman spectroscopic characterization of some commercially 
available carbon black materials, Carbon 33 (1995) 1561-1565.

[37] S. Sze, Raman spectroscopic characterization of carbonaceous aerosols, Atmos. Environ. 35 (2001) 561-568.

[38] B. Dippel, H. Jander, J. Heintzenberg, NIR FT Raman spectroscopic study of flame soot, Phys. Chem. Chem. Phys. 1 (1999) 4707-4712.

[39] M.J. Madito, A. Bello, J.K. Dangbegnon, C.J. Oliphant, W.A. Jordaan, T.M. Masikhwa, D. Y. Momodu, N. Manyala., Raman analysis of bilayer graphene film prepared on commercial $\mathrm{Cu}(0.5$ at\% Ni) foil, J. Raman Spectrosc. (2015).

[40] Q. Abbas, D. Pajak, E. Frąckowiak, F. Béguin, Effect of binder on the performance of carbon/carbon symmetric capacitors in salt aqueous electrolyte, Electrochim. Acta. 140 (2014) 132-138.

[41] W.G. Pell, B.E. Conway, N. Marincic, Analysis of non-uniform charge/discharge and rate effects in porous carbon capacitors containing sub-optimal electrolyte concentrations, J. Electroanal. Chem. 491 (2000) 9-21.

[42] R. Kötz, M. Carlen, Principles and applications of electrochemical capacitors, Electrochim. Acta. 45 (2000) 2483-2498.

[43] J. Luo, H.D. Jang, J. Huang, Effect of sheet morphology on the scalability of graphene-based ultracapacitors, ACS Nano. 7 (2013) 1464-1471. 